\title{
Updates in Pediatric Resuscitation: Recent Advances and Current Concepts
}

\author{
Alexander F. Pitfield · Shelina Jamal • \\ Niranjan Kissoon
}

Published online: 19 December 2012

(C) Springer Science + Business Media New York 2012

\begin{abstract}
Pediatric cardiac arrest is a rare condition generally associated with a low likelihood of neurologically intact survival. In pediatrics, cardiopulmonary resuscitation (CPR) has traditionally focused on the management of the airway and restoration of adequate ventilation. Recent advances in the understanding of resuscitation physiology and technology have dramatically changed the landscape for pediatric resuscitation. As is often the case, old habits die hard-global adoption of these newer concepts has been slow and incomplete. This article summarizes the recent advances in CPR, addressing both resuscitative and post-resuscitative care.
\end{abstract}

Keywords Critical illness - Children - Resuscitation · Cardiopulmonary resuscitation $\cdot$ Management .

Extracorporeal CPR

\section{Introduction}

The 2010 American Heart Association (AHA) guidelines for advanced cardiac life support changed the way practitioners think of cardiopulmonary resuscitation (CPR). The move from $\mathrm{ABC}$ (airway, breathing, circulation) to $\mathrm{CAB}$ intended to shift rescuers' focus from the technically difficult management of the airway and ventilation to the more straightforward provision of chest compressions. The

\footnotetext{
A. F. Pitfield · S. Jamal · N. Kissoon $(\bowtie)$

Critical Care Division, Department of Pediatrics, British

Columbia's Children's Hospital, Suite B245-4480 Oak Street,

Vancouver, BC V6H 3V4, Canada

e-mail: nkissoon@cw.bc.ca

S. Jamal

Division of Cardiovascular Intensive Care, Department of

Cardiology, Boston Children's Hospital, Boston, MA, USA
}

change has not only permitted the simplification of training for lay rescuers, but also shifted our priorities in resuscitative scenarios. The paramount importance of chest compressions has been emphasized as never before.

With such great change, we find ourselves questioning our pediatric resuscitative practices in general. Medications, equipment, technologies and others are being critically examined. While pediatric data is sparse, we are unable to ignore the emergence of large adult studies supporting changes in practice regarding all of these. The randomized controlled trial data in adults is often supported by retrospective cohort studies in children.

The changes in the 2010 guidelines secured the belief that resuscitative medicine is a changing field, with new technologies and techniques available to medical practitioners. This review provides an assessment of current pediatric concepts regarding CPR.

\section{Initiation and Quality of Cardiopulmonary Resuscitation}

Initiation of CPR

Better outcomes rely on rapid diagnosis of cardiac arrest and immediate institution of CPR. However, rapid diagnosis of cardiac arrest can be surprisingly difficult, even for healthcare professionals. For instance, Tibballs et al. [1•] demonstrated that pulse checks performed by pediatric healthcare providers were inaccurate; rescuers misdiagnosed cardiac arrest in $22 \%$ of cases, would have withheld cardiac compressions in $14 \%$ of cases where they were indicated, and would have performed unnecessary cardiac compressions in $36 \%$ of cases. Their study also reported that brachial palpation was slightly more reliable than 
femoral palpation. Similar to other studies, they concluded that pulse palpation is unreliable in diagnosing pediatric cardiac arrest. Moreover, a similar study showed that rescuers took too long to make a decision regarding presence or absence of a pulse (mean time $20 \pm 17 \mathrm{~s}$ ) [2]. They also took a mean time of $30 \pm 19 \mathrm{~s}$ to diagnose the absence of a pulse, and $13 \pm 13 \mathrm{~s}$ to diagnose the presence of a pulse. Experienced doctors (those with training in acute care disciplines) were the fastest and most accurate in their determinations. Cognizant of these findings, the recently released AHA pediatric resuscitation guidelines state that lay rescuers should initiate CPR if the victim is unresponsive, not breathing normally, and without signs of life. In infants and children with signs of life, healthcare providers should begin CPR unless they can definitely palpate a pulse within $10 \mathrm{~s}[3 \bullet \bullet]$.

The recommendations encouraging earlier administration of CPR are reasonable because of the low risk associated with chest compressions. A 2006 review of 923 children who had CPR found three children who sustained rib fractures as a result of chest compressions; all were anterior (two mid-clavicular and one costo-chondral) and none were associated with long-term medical sequelae [4]. The infrequency of rib fractures in this population relates to the flexibility of the chest wall. Additional risks include pneumothorax from fractured rib and/or abdominal visceral organ rupture with inappropriately positioned compressions, however none of these have been reported in children.

\section{CPR Sequence-ABC to $\mathrm{CAB}$}

The 2010 AHA guidelines sought to minimize delays in initiating cardiac compressions by revising the sequence from $\mathrm{ABC}$ (airway, breathing, compressions) to $\mathrm{CAB}$ (compressions, airway, breathing), thereby reiterating the importance of cardiac compressions. This change in sequence is endorsed in infant, child and adult resuscitation despite major differences in etiology (dysrhythmia in adults, asphyxia in children). Across all age groups, the most common cause of cardiac arrest is ventricular fibrillation/ventricular tachycardia (VF/VT). The adoption of the CAB sequence in pediatrics simplifies the teaching of resuscitation skills.

Importantly in pediatrics, data shows that the practice is not necessarily harmful in asphyxial cardiac arrest. In pediatric CPR, the shorter the period of time elapsed between collapse and the first chest compression is a reliable predictor of sustained return of spontaneous circulation [5•]. Shorter times may be facilitated by using the $\mathrm{CAB}$ sequence rather than the $\mathrm{ABC}$ sequence, as evidenced by a randomized trial comparing times of intervention during pediatric $\mathrm{CPR}$ maneuvers using $\mathrm{ABC}$ and $\mathrm{CAB}$ sequences in a simulation setting [6]. The $\mathrm{CAB}$ sequence prompted quicker recognition of respiratory or cardiac arrest and quicker initiation of CPR. In the cardiac arrest scenario, the $\mathrm{CAB}$ sequence resulted in cardiac compressions starting earlier compared with the $\mathrm{ABC}$ sequence (19 \pm 2 vs. $43 \pm 5$ s, respectively). Interestingly, assisted ventilations started earlier in the respiratory arrest scenario when the $\mathrm{CAB}$ sequence was used instead of $\mathrm{ABC}$ sequence (19 \pm 1 vs. $23 \pm 3$ s respectively).

Conversely, compression-only CPR has not been shown to be effective in young patients. A 2010 meta-analysis of adults with out-of-hospital cardiac arrest showed that compression-only CPR was associated with improved chance of survival compared with standard CPR [7]. However, a 2011 observational study of approximately 40,000 patients comparing the effectiveness of compression only CPR with conventional CPR demonstrated that both 1-month survival and neurologically favorable 1-month survival rates after witnessed out-of-hospital cardiac arrest were higher in the conventional CPR group [8••]. Not surprisingly, the benefit of conventional CPR over compression only CPR was significantly greater at younger ages (0-19 years) for arrests primarily of noncardiac origin, but similar at all ages for arrests primarily of cardiac origin and all cases combined. This reflects the increased number of respiratory arrests leading to cardiac arrest in the pediatric population. No studies have been done exclusively looking at outcomes of compression only $\mathrm{CPR}$ in the pediatric population.

\section{Minimizing Interruptions of Compressions}

The present recommendations discourage frequent interruption of chest compressions because of the deleterious effect on coronary perfusion. Experimental models have shown that coronary perfusion pressure is a major determinant of survival from cardiac arrest [9]. Animal studies show that coronary perfusion pressure rapidly declines when chest compressions are interrupted. Once compressions are resumed, several chest compressions are needed to restore coronary perfusion pressure to pre-interruption levels. Thus, frequent interruptions of chest compressions prolong the duration of low coronary perfusion pressure and flow and reduce the mean coronary artery perfusion pressure. Adult studies have since demonstrated that these interruptions prolong the time to ROSC [10].

\section{Assessment of CPR Quality}

\section{Chest Compressions}

The goal of effective CPR is to optimize coronary and cerebral perfusion and blood flow to critical organs during this low-flow phase. The emphasis in this phase is the implementation of effective chest compressions (push hard, 
push fast, allow full chest recoil, minimize interruptions, and do not over-ventilate). The depth of compressions should be at least one-third of the chest's antero-posterior diameter. The recommended compression rate remains constant for all ages (100 compressions per minute). It is recommended that rescuers should change compressors every $2 \mathrm{~min}$ prior to onset of rescuer fatigue. A one- or two-hand technique is acceptable when performing chest compressions in children.

The survival benefit of high-quality CPR (Q-CPR) is well documented, but little objective data exist regarding actual CPR quality during cardiac arrest. Approximately 5 years ago, quantitative CPR monitoring systems equipped with a force transducer and accelerometer were developed and deployed during adult arrest. These Q-CPR devices can record objective data on force, depth and timing, rate of compressions, and can provide real-time automated audio and visual directive and corrective feedback during actual cardiac arrests through interface with defibrillator systems. Subsequent studies have demonstrated improvement in CPR quality when these devices are employed [11].

Little has been published on quality of pediatric CPR. In 2009, Sutton used a Q-CPR device to evaluate rate, depth, and quality of chest compressions and ventilations delivered to 20 pediatric patients during in-hospital cardiac arrest. Deficiencies were demonstrated in ability to attain published guideline targets for quality of CPR. Chest compressions were frequently performed at an inappropriate rate (primarily too slow) and/or depth (primarily too shallow). This was the first study that demonstrated that parameters of CPR quality could be tracked and reported during pediatric CPR as a real-time quality improvement tool [12•].

A widely available and practical approach to providing feedback during CPR is continuous capnography or capnometry. Monitoring of end-tidal carbon dioxide (ETCO2) levels in cardiac arrest was first described over 30 years ago [13]. A drop in ETCO2 indicated inadequate compression efficacy, and signaled the need for more vigorous compressions or a new provider to replace a fatigued colleague and perform chest compressions. Nearly 10 years later, ETCO2 levels were demonstrated in an animal model to fall immediately at the onset of cardiac arrest, increase immediately with chest compressions, provide a linear correlation with cardiac index, predict successful resuscitation, and allow detection of ROSC when a sudden increase in the ETCO2 level occurred [14]. These results have since been reproduced in multiple human and animal studies. If the ETCO 2 is consistently $\leq 15 \mathrm{mmHg}$, it is reasonable to focus efforts on improving the quality of chest compressions and avoiding excessive ventilation.

\section{Ventilation}

Although harmful, excessive ventilation during CPR is a common occurrence. Increased ventilation rate in animals is associated with significantly lower coronary perfusion pressures [15]. In a series of 72 pediatric simulated medical emergencies, hyperventilation was present in every mock code reviewed, with a mean rate of bag-valve mask ventilation of $40 \pm 12$ breaths per minute [16]. The 2010 AHA guidelines state that while there is insufficient data to identify the optimal tidal volume or respiratory rate, a reduction in minute ventilation to less than baseline for age is reasonable to maintain an adequate ventilation:perfusion ratio during $\mathrm{CPR}$ while avoiding the harmful effects of excessive ventilation.

\section{Resuscitation Training}

Cardiopulmonary resuscitation teaching has classically consisted of infrequent and intensive training courses. Many healthcare providers receive initial CPR certification following a 1- or 2-day course, and maintain that certification through yearly refresher courses. A growing body of evidence demonstrates that this approach not only results in suboptimal skill acquisition, but also inadequate skill retention 3-6 months after initial training [17-19]. Newer techniques to improve skill retention and performance are described here.

\section{Booster Training}

Low-dose, high-frequency CPR training ("booster training") is a technique to facilitate skill retention described by Sutton and colleagues. This group has described significant improvements in CPR quality following a 2-min CPR "booster"; either instructor led or automated using a commercially available system [20••, 21]. These sessions focus on the basics of good chest compressions: adequate rate and depth, minimal interruptions, and allowing complete chest recoil. Practitioners with quarterly exposure to these 2-min sessions showed not only good retention of skills, but also gradual improvement in their baseline CPR quality. These sessions can be performed during normal work hours, making them an attractive option in a resource challenged environment.

\section{Post-resuscitation Debriefing}

The 2010 AHA guidelines formally endorse the use of debriefing to improve the quality of simulation-based CPR education. Traditional CPR training has relied heavily on feedback, during which the instructor relays observations to the learner. With increasing use of high-fidelity simulation in resuscitation courses, skill in post-scenario debriefing becomes necessary. Debriefing may be defined as "facilitated or guided reflection in the cycle of 
experiential learning" [22]. This newer approach improves outcomes for CPR learners, and consequently a debriefing module has been added to the most recent Pediatric Advanced Life Support instructor support materials [23].

In actual resuscitation situations, post-resuscitation quantitative debriefing may also play an important role. Chest compression depth and rate improved significantly for medical residents in a prospective interventional trial using a historical control [24]. Post-resuscitation quantitative debriefing has recently been reported in pediatrics, where practitioners were debriefed on eight items observed during the resuscitation (chest compression quality, noflow time, defibrillation, ETCO2, arrest physiology, rhythm management, medication usage, and communication). These debriefs were well received by practitioners, demonstrating that in situ debriefing can be easily performed [25]. Coupled with the knowledge that such debriefs improve the quality of CPR delivered by a resuscitation team, this information should encourage leaders to debrief with their team, at least quantitatively, following every resuscitation.

\section{Advances in Resuscitation Strategies}

\section{Standard Cardiac Resuscitation Medications}

\section{Epinephrine}

Epinephrine is the drug of choice in the management of cardiac arrest. Its vasoconstrictive properties have been demonstrated to improve coronary and cerebral perfusion during cardiac arrest. A 2009 study of adult out-of-hospital cardiac arrest, which randomized patients to receive epinephrine or placebo, demonstrated faster time to ROSC in the epinephrine group; however, this did not translate to improved survival to hospital discharge [26]. A systematic review of 53 articles demonstrated that epinephrine is associated with improvement in short-term survival outcomes when compared with placebo in adults, although no long-term survival benefit was demonstrated [27]. No studies have compared epinephrine with placebo in the pediatric population.

Regarding the appropriate dose of epinephrine during $\mathrm{CPR}$, a single randomized controlled trial in pediatrics compared the use of standard dose $(0.01 \mathrm{mg} / \mathrm{kg})$ versus high dose $(0.1 \mathrm{mg} / \mathrm{kg})$ epinephrine [28]. No difference in rate of survival was noted amongst the 68 patients enrolled. However, a trend did emerge amongst those patients with asphyxial cardiac arrest suggesting that high-dose therapy was harmful. As a result of these findings, subsequent resuscitation guidelines have suggested standard dose epinephrine $(0.01 \mathrm{mg} / \mathrm{kg})$ to be used throughout resuscitation.

\section{Atropine}

Bradycardic arrest in children is most often due to hypoxia. The 2010 AHA guidelines recommended administration of epinephrine if bradycardia persists despite oxygen administration and/or assisted ventilation. The use of atropine is limited to primary conduction problems, increased vagal tone and cholinergic toxicity. The adult resuscitation guidelines recommend atropine for symptomatic bradycardia, followed by pacing and/or epinephrine as secondline interventions. This difference reflects the different etiology for bradycardic arrest in adults (primary cardiac origin). A concern with administration of atropine in children is the risk of paradoxical bradycardia. To date no studies have compared atropine with epinephrine in the management of bradycardic arrest in children.

\section{Sodium Bicarbonate}

No randomized controlled trials have assessed the use of sodium bicarbonate in the management of pediatric cardiac arrest. A multicenter, retrospective study of in-hospital pediatric cardiac arrest reported that sodium bicarbonate administration was independently associated with increased mortality [29]. The reason for this is unclear, but it may be due to the carbon dioxide generated by bicarbonate administration, which may not be cleared despite adequate ventilation during a low-flow resuscitative state. It is believed that the resulting respiratory acidosis can potentially worsen existing metabolic acidosis and have deleterious effects on myocardial performance [30]. The 2010 AHA guidelines reiterate the lack of evidence for routine use of sodium bicarbonate in pediatric cardiac arrest.

\section{Calcium}

No randomized controlled trials have assessed the use of calcium in the management of pediatric cardiac arrest. A multicenter, retrospective study of in-hospital pediatric cardiac arrest reported that calcium administration was independently associated with increased mortality [29]. Increased cytoplasmic calcium concentration activates intracellular enzyme systems resulting in cellular necrosis [31]. As early as 2000 the recommendation has been that administration of calcium in the cardiac arrest setting be limited to documented hyocalcemia, hyperkalemia, and calcium channel blocker overdose. Nonetheless, during in-hospital pediatric cardiac arrests calcium use is reported in $45 \%$ of events [32]. Its use was also associated with decreased survival to discharge. Calcium use should therefore be limited to known cases of hypocalcemia, hyperkalemia, hypermagnesemia, or calcium channel blocker toxicity. 


\section{Vasopressin}

The 2010 AHA guidelines support the use of a single dose of vasopressin (40 $\mathrm{U}$, to replace either the first or second dose of epinephrine) in the management of adult cardiac arrest. A retrospective registry review in 2009 showed an association between vasopressin use and worse ROSC [33]. Pediatric use of vasopressin appears to be practitioner dependent, with occasional use during in-hospital cardiac arrest (primarily in the intensive care unit). Its use in pediatrics has not been well studied, and the 2010 AHA guidelines do not recommend its routine use in pediatric cardiac arrest.

\section{Extracorporeal CPR (eCPR)}

Over the past decade, extracorporeal life support (ECLS) has emerged as one of the defining life support technologies available to critical care practitioners. Initially used primarily for neonatal respiratory failure, it has become a widespread advanced support modality for use in severe cardio-respiratory failure in all age groups. Recognizing that $40-50 \%$ of in-hospital pediatric cardiac arrest victims will not be successfully resuscitated by PALS guided resuscitation techniques [29, 34], many centers worldwide have established programs to facilitate rapid deployment of ECLS in this population.

If adequate cerebral perfusion can be maintained until extracorporeal circulation is established, it is possible to achieve intact neurological outcome. The overall outcome is largely reliant on the early and aggressive performance of chest compressions, with minimal interruptions. A wellrehearsed mechanism for rapid deployment of ECLS must exist for eCPR to be a feasible option. Typically this involves limiting use of these resources to cases of witnessed in-hospital cardiac arrest, although our center has achieved excellent outcomes in two patients suffering witnessed out-of-hospital cardiac arrest. To ensure that both timely PALS resuscitation is provided and ECLS is expeditiously initiated, we recommend that one physician team leader coordinates standard resuscitative interventions while a second physician team leader coordinates the deployment of ECLS.

Outcome data for eCPR is limited. For neonatal patients, the Extracorporeal Life Support Organization 2012 registry data shows that $63 \%$ of patients could be weaned from ECLS, with an overall survival to discharge or transfer of $38 \%$. For pediatric patients, $53 \%$ survived ECLS and $40 \%$ survived to discharge or transfer [35]. This data is difficult to interpret, because criteria for eCPR are variable. However recognizing that most patients who have not achieved ROSC in 20-40 min will die (approximately the time required for ECLS cannulation), the survival numbers in the ELSO database are impressive. A recent prospective observational study in adults also demonstrated a survival benefit from eCPR [36]. The quality of neurological outcome following eCPR is not well described, and clearly this requires further study.

While epinephrine is the drug of choice in CPR, postresuscitation epinephrine toxicity can impair the ability to provide adequate extracorporeal blood flow, especially when the ECLS circuit uses a centrifugal blood pump. These pumps are afterload dependent, and the elevated systemic vascular resistance increases afterload thereby decreasing pump flow for any given rotational frequency. Therefore those patients with clinically significant elevations of systemic vascular resistance following initiation of extracorporeal support commonly require systemic vasodilators including milrinone and sodium nitroprusside.

\section{Post-resuscitation Management}

\section{Therapeutic Hypothermia}

The use of induced hypothermia for neuro-protection after pediatric cardiac arrest has been of long-standing interest. Reports of impressive neurological recovery following prolonged cold water drowning initially created interest in therapeutic hypothermia for other indications [37]. It was widely used as a neuro-protective therapy following pediatric drowning through the 1970s [38], and was used following asphyxial cardiac arrest into the 1980s [38, 39]. The therapy was subsequently largely abandoned due to difficulties with reliable and safe application, coupled with unclear benefits [40]. Resurgence of interest in perinatal medicine led the way for the major adult studies demonstrating both safety and efficacy of induced hypothermia following ventricular fibrillation cardiac arrest. Induced hypothermia has become an established neuro-protective measure for adult victims of VF/VT cardiac arrest since its use has been demonstrated to reduce mortality and improve neurological outcome [41, 42]. In addition, the 2010 AHA guidelines advocate use for PEA and asystolic cardiac arrest in adults [43].

Current definitive pediatric evidence to guide practice does not exist. It has been demonstrated that therapeutic hypothermia can be employed safely in pediatrics [44]. The ongoing THAPCA trial, which will be the first randomized controlled trial studying outcomes for therapeutic hypothermia after in- and out-of-hospital cardiac arrest in children may offer some direction. Despite the lack of evidence in pediatrics, many centers have adopted the practice based on the definitive adult and perinatal literature [45, 46]. Indications for use vary widely, although most centers include the cohort of children who have 
suffered sudden cardiac arrest of non-respiratory origin in the inclusion group.

When used, therapeutic hypothermia should be initiated as soon as possible after arrest, potentially even in the prehospital environment. The target temperature is $32-34{ }^{\circ} \mathrm{C}$, which is maintained for $24-48 \mathrm{~h}$. Subsequent care is variable: rewarming typically occurs at a rate of $0.5^{\circ} \mathrm{C}$ every $2 \mathrm{~h}$, and normothermia is typically maintained for 2-4 additional days.

In summary, therapeutic hypothermia can be safely administered to pediatric patients. It has not been clearly demonstrated to improve outcomes following pediatric cardiac arrest. Based on adult and perinatal evidence, however, it is reasonable to consider this therapy in the pediatric population.

\section{Conclusion}

Pediatric resuscitative practices have historically focused on the management of airway and ventilation. Advances in the understanding of adult resuscitative care have set the stage for pediatricians to re-examine this dogma. In addition, adult studies have allowed us to change our approach to medical therapy during CPR and post-resuscitative care. Despite the current lack of definitive pediatric evidence, our search for answers to questions raised about our current practices will improve the future quality of care delivered to our patients.

Conflict of interest No potential conflicts of interest relevant to this article were reported.

\section{References}

Papers of particular interest, published recently, have been highlighted as:

- Of importance

-• Of major importance

1. - Tibballs J, Russell P. Reliability of pulse palpation by healthcare personnel to diagnose pediatric cardiac arrest. Resuscitation. 2009;80:61-4. An alarming report demonstrating healthcare practitioners' difficulty with diagnosing cardiac arrest in children. Clearly supportive of the move towards initiation of CPR based on easily appreciable clinical signs (unresponsive, not breathing normally, no signs of life).

2. Tibballs J, Weeranatna C. The influence of time on the accuracy of healthcare personnel to diagnose paediatric cardiac arrest by pulse palpation. Resuscitation. 2010;81:671-5.

3. •- De Caen AR, Kleinman ME, Chameides L et al. Pediatric basic and advanced life support; 2010 International consensus on cardiopulmonary resuscitation and emergency cardiovascular care science with treatment recommendations. Resuscitation. 2010;81S:e213-59. This comprehensive description of resuscitative algorithms for pediatrics is required reading for any pediatric healthcare practitioner.

4. Maguire S, Mann M, John N, et al. Does cardiopulmonary resuscitation cause rib fractures in children?. A systematic review. Child Abuse Negl. 2006;30:739-51.

5. - Li CJ, Kung CT, Liu BM et al. Factors associated with sustained return of spontaneous circulation in children after out-of-hospital cardiac arrest of noncardiac origin. Am $J$ Emerg Med. 2010;28:310-317. Demonstrates the importance of early CPR in children, and supports adoption of the $C-A-B$ resuscitation sequence in this population.

6. Lubrano R, Cecchetti C, Bellelli E et al. Comparison of times of intervention during pediatric $C P R$ maneuvers using $A B C$ and $C A B$ sequences: a randomized trial. Resuscitation. 2012;83:1473-7.

7. Hupfl M, Selig HF, Nagele P. Chest-compression-only versus standard cardiopulmonary resuscitation: a meta-analysis. Lancet. 2010;376:1552-7.

8. - Ogawa T, Akahane M, Koike S et al. Outcomes of chest compression only CPR versus conventional CPR conducted by lay people in patients with out of hospital cardiopulmonary arrest witnessed by bystanders: nationwide population based observational study. BMJ. 2011;342:c7106. This large observational study demonstrates the ongoing importance of ventilatory management in pediatric cardiac arrest. For the time being, this information may put the move to compression-only CPR on hold.

9. Kern KB, Ewy GA, Voorhees WD, et al. Myocardial perfusion pressure: a predictor of 24-hour survival during prolonged cardiac arrest in dogs. Resuscitation. 1988;16:241-50.

10. Berg RA, Sanders AB, Kern KB, et al. Adverse hemodynamic effects of interrupting chest compression for rescue breathing during CPR for VF cardiac arrest. Circulation. 2001;104:2465-70.

11. Abella BS, Edelson DP, Kim S, et al. CPR quality improvement during in-hospital cardiac arrest using a real-time audiovisual feedback system. Resuscitation. 2001;73:54-61.

12. - Sutton RM, Maltese MR, Niles D et al. Quantitative analysis of CPR quality during in-hospital resuscitation of older children and adolescents. Pediatrics. 2009;124:494-9. Despite best efforts, quality of in-hospital CPR remains low. CPR quality can be tracked in the hospital setting, and provides a valuable tool to facilitate the improvement of chest compression technique.

13. Kalenda $Z$. The capnogram as a guide to the efficacy of cardiac massage. Resuscitation. 1978;6:259-63.

14. Gudipati CV, Weil MH, Bisera J, et al. Expired carbon dioxide: a non-invasive monitor of cardiopulmonary resuscitation. Circulation. 1988;77:234-9.

15. Aufderheide TP, Lurie KG. Death by hyperventilation: a common and life-threatening problem during cardiopulmonary resuscitation. Crit Care Med. 2004;32:S345-51.

16. Niebauer JM, White ML, Zinkan JL, et al. Hyperventilation in pediatric resuscitation: performance in simulated pediatric medical emergencies. Pediatrics. 2011;128:e1195-200.

17. Hamilton R. Nurses' knowledge and skill retention following cardiopulmonary resuscitation training: a review of the literature. J Adv Nurs. 2005;51(3):288-97.

18. Kaye W, Mancini ME. Retention of cardiopulmonary resuscitation skills by physicians, registered nurses, and the general public. Crit Care Med. 1986;14(7):620-2.

19. Mancini ME, Kaye W. The effect of time since training on house officers' retention of cardiopulmonary resuscitation skills. Am J Emerg Med. 1985;3(1):31-2.

20. • Sutton RM, Niles D, Meaney PA et al. "Booster" training: evaluation of instructor-led bedside cardiopulmonary resuscitation skill training and automated corrective feedback to improve cardiopulmonary resuscitation compliance of Pediatric Basic Life Support providers during simulated cardiac arrest. Pediatr Crit Care Med. 2011;12(3):e116-21. A clear demonstration that 
hospital based CPR quality improvement can be achieved simply, quickly, and at a low cost. The baseline data is consistent with other reports describing the low overall quality of CPR technique amongst healthcare providers.

21. Sutton RM, Niles D, Meaney PA, et al. Low-dose, high-frequency CPR training improves skill retention of in-hospital pediatric providers. Pediatrics. 2011;128(1):e145-51.

22. Fanning RM, Gaba DM. The role of debriefing in simulationbased learning. Simul Healthc. 2007;2:115-25.

23. Cheng A, Rodgers DL, van der Jagt E, et al. Evolution of the Pediatric Advanced Life Support course: enhanced learning with a new debriefing tool and web-based module for Pediatric Advanced Life Support instructors. Pediatr Crit Care Med. 2012;13(5):589-95.

24. Edelson DP, Litzinger B, Arora V, et al. Improving in-hospital cardiac arrest process and outcomes with performance debriefing. Arch Intern Med. 2008;168:1063-9.

25. Zebuhr C, Sutton RM, Morrison W, et al. Evaluation of quantitative debriefing after pediatric cardiac arrest. Resuscitation. 2012;83:1124-8.

26. Olasveengen TM, Sunde K, Brunborg C, et al. Intravenous drug administration during out-of-hospital cardiac arrest: a randomized trial. JAMA. 2009;302:2222-9.

27. Larabee TM, Liu KY, Campbell JA, et al. Vasopressors in cardiac arrest: a systematic review. Resuscitation. 2012;83(8):932-9.

28. Perondi MB, Reis AG, Paiva EF, et al. A comparison of highdose and standard-dose epinephrine in children with cardiac arrest. N Engl J Med. 2004;350:1722-30.

29. Meert KL, Donaldson A, Nadkarni V, et al. Multicenter cohort study of in-hospital pediatric cardiac arrest. Pediatr Crit Care Med. 2009;10(5):544-53.

30. Aschner JL, Poland RL. Sodium bicarbonate: basically useless therapy. Pediatrics. 2008;122(4):831-5.

31. Wetzel R. Calcium: a double-edged sword. Pediatr Crit Care Med. 2007;8(3):300-1.

32. Srinivasan V, Morris MC, Helfaer MA, et al. Calcium use during in-hospital pediatric cardiopulmonary resuscitation: a report from the NRCPR. Pediatrics. 2008;121(5):e1144-51.

33. Duncan JM, Meaney P, Simpson P, et al. Vasopressin for inhospital pediatric cardiac arrest: results from the American Heart Association National Registry of Cardiopulmonary Resuscitation. Pediatr Crit Care Med. 2009;10(2):191-5.
34. Berg MD, Nadkarni VM, Zuercher M, Berg RA. In-hospital pediatric cardiac arrest. Pediatr Clin N Am. 2008;55(3):589-604.

35. Extracorporeal Life Support Organization. ECLS Registry Report: International Summary. ELSO. Jan 2012.

36. Chen YS, Lin JW, Yu HY, et al. Cardiopulmonary resuscitation with assisted extracorporeal life-support versus conventional cardiopulmonary resuscitation in adults with in-hospital cardiac arrest: an observational study and propensity analysis. Lancet. 2008;372:554-61. doi:10.1016/S0140-6736(08)60958-7.

37. Siebke H, Rod T, Breivik H. Survival after 40 minutes submersion without cerebral sequelae. Lancet. 1975;1(7919):1275-7.

38. Conn AW, Edmonds JF, Barker GA. Cerebral resuscitation in near-drowning. Pediatr Clin N Am. 1979;26:691-701.

39. Nussbaum E, Maggi JC. Pentobarbital therapy does not improve neurologic outcome in nearly drowned, flaccid-comatose children. Pediatrics. 1988;81:630-4.

40. Bohn DJ, Biggar WD, Smith CR, et al. Influence of hypothermia, barbiturate therapy, and intracranial pressure monitoring on morbidity and mortality after near-drowning. Crit Care Med. 1986;14:529-34.

41. The Hypothermia after Cardiac Arrest Study Group. Mild therapeutic hypothermia to improve the neurologic outcome after cardiac arrest. N Engl J Med. 2002;346(8):549-56. This seminal paper prompted modern interest in therapeutic hypothermia after cardiac arrest.

42. Bernard SA, Gray TW, Buist MD, et al. Treatment of comatose survivors of out-of-hospital cardiac arrest with induced hypothermia. N Engl J Med. 2002;346(8):557-63.

43. Peberdy MA, Callaway CW, Neumar RW, et al. Part 9: postcardiac arrest care. Circulation. 2010;122(suppl):S768-86.

44. Fink EL, Clark RS, Kochanek PM, et al. A tertiary care center's experience with therapeutic hypothermia after pediatric cardiac arrest. Pediatr Crit Care Med. 2010;11:66-74.

45. Kochanek PM, Fink EL, Bell MJ, et al. Therapeutic hypothermia: applications in pediatric cardiac arrest. J Neurotrauma. 2010;26:421-7.

46. Berg MD. Pediatric in-hospital cardiac arrest and therapeutic hypothermia: where we are and where we are going. Pediatr Crit Care Med. 2009;10(5):601-2. 